\title{
3D Imaging of Nanoalloy Catalysts at Atomic Resolution
}

\author{
Jihan Zhou ${ }^{1}$, Yongsoo Yang $^{1}$, Zipeng Zhao ${ }^{2}$, Colin Ophus ${ }^{3}$, Peter Ercius $^{3}$, Yu Huang ${ }^{2}$ and Jianwei Miao $^{1}$ \\ 1. Department of Physics \& Astronomy and California NanoSystems Institute, University of California, \\ Los Angeles, CA 90095, USA. \\ 2. Department of Materials Science \& Engineering and California NanoSystems Institute, University of \\ California, Los Angeles, CA 90095, USA. \\ 3. National Center for Electron Microscopy, Molecular Foundry, Lawrence Berkeley National \\ Laboratory, Berkeley, California, USA.
}

Metal catalysts such as $\mathrm{Pt}, \mathrm{Pd}, \mathrm{Cu}$, and $\mathrm{Ni}$ are critical components in many applications ranging from fuel cells in automobiles to mobile power generation. Recent studies have shown that introducing earthabundant metals such as $\mathrm{Ni}$ and $\mathrm{Cu}$ into $\mathrm{Pt}$ can not only significantly enhance the oxygen reduction reaction (ORR) activity of the catalysts, but also lower the cost [1, 2]. Among these nanoalloys, bimetallic platinum-nickel (PtNi) nanostructures represent an emerging class of candidates for ORR catalyst in fuel cells. More recently, a new nanoalloy catalyst of carbon-supported NiPt with Mo doped on the surface (PtNi-Mo) has been reported, demonstrating 80-fold enhancements compared with commercial Pt-C catalyst [3]. To understand the structure and performance of these nanoalloy catalysts, it is essential to fully characterize the surface structure of these nanoalloy catalysts in 3D with atomic resolution. An ideal method to achieve this challenging goal is atomic electron tomography (AET) [4], which retrieves 3D atomic structure information from a tilt series of high-resolution 2D images. In recent years, AET has been used to image the 3D structure of grain boundaries, stacking faults, and the core structure of edge and screw dislocations at atomic resolution [5, 6]. Furthermore, the combination of AET and atom tracing algorithms has enabled the determination of the coordinates of individual atoms and point defects in materials with a 3D precision of $\sim 19 \mathrm{pm}$, allowing direct measurements of 3D atomic displacements and the full strain tensor [7]. More recently, the 3D coordinates of more than 23,000 atoms in an FePt nanoparticle have been determined by AET to correlate chemical order/disorder and crystal defects with material properties at the single-atom level [8]. Here, we apply AET to probe the 3D surface atomic structure of PtNi-Mo nanoalloy catalysts. By using the TEAM microscope at the National Center for Electron Microscopy at LBNL, we have acquired several tomographic tilt series from PtNi-Mo nanoalloys. After post processing and centre of mass alignment of the tilt series, we computed high-quality 3D reconstructions by using a Generalized Fourier Iterative Reconstruction (GENFIRE) algorithm [8]. Volume-renderings of a representative 3D reconstruction show individual atoms at different orientations (Fig. 1). The individual Pt, Ni and Mo atoms can be distinguished based on the intensity contrast. Figure 2 shows three perpendicular $1.7 \AA$ thick central slices of the atomic resolution reconstruction, where red and blue arrows indicate $\mathrm{Pt}$ and $\mathrm{Ni}$ atoms, respectively. With further improvement of the tilt series alignment, denoising and tilt angle refinement, we expect the full determination of the 3D atomic structure of PtNi-Mo nanoalloys, including precise atomic coordinates and accurate classification of $\mathrm{Pt}, \mathrm{Ni}$, and $\mathrm{Mo}$ atoms. Coupled with first principles calculations, the full surface oxygen binding energy map can be derived from the 3D atomic structure, providing essential information to precisely tailor and optimize the catalytic behavior for various applications [9].

\section{References:}

[1] M.K. Debe, Nature 486, (2012), p. 43-51.

[2] V. R. Stamenkovic, et al., Nat. Mater. 6, (2007), p. 241-247. 
[3] X.Q. Huang et al., Science 348, (2015), p. 1230-1234.

[4] J. Miao et al., Science 353, (2016), p. aaf2157.

[5] Scott, M. C. et al. Nature 483, (2012), p. 444-447.

[6] Chen, C. C. et al. Nature 496, (2013), p. 74-77.

[7] R. Xu et al., Nat. Mater. 14 (2015), p. 1099-1103.

[8] Y. Yang et al., Nature 542 (2017), p. 75-79.

[9] This work was supported by NSF (DMR-1548924 and DMR-1437263), DOE BES (DE-SC0010378). The ADF-STEM imaging with TEAM 05 were performed at the Molecular Foundry, which is supported by the Office of Science, Office of Basic Energy Sciences of the U.S. DOE under Contract No. DEAC02--05CH11231.
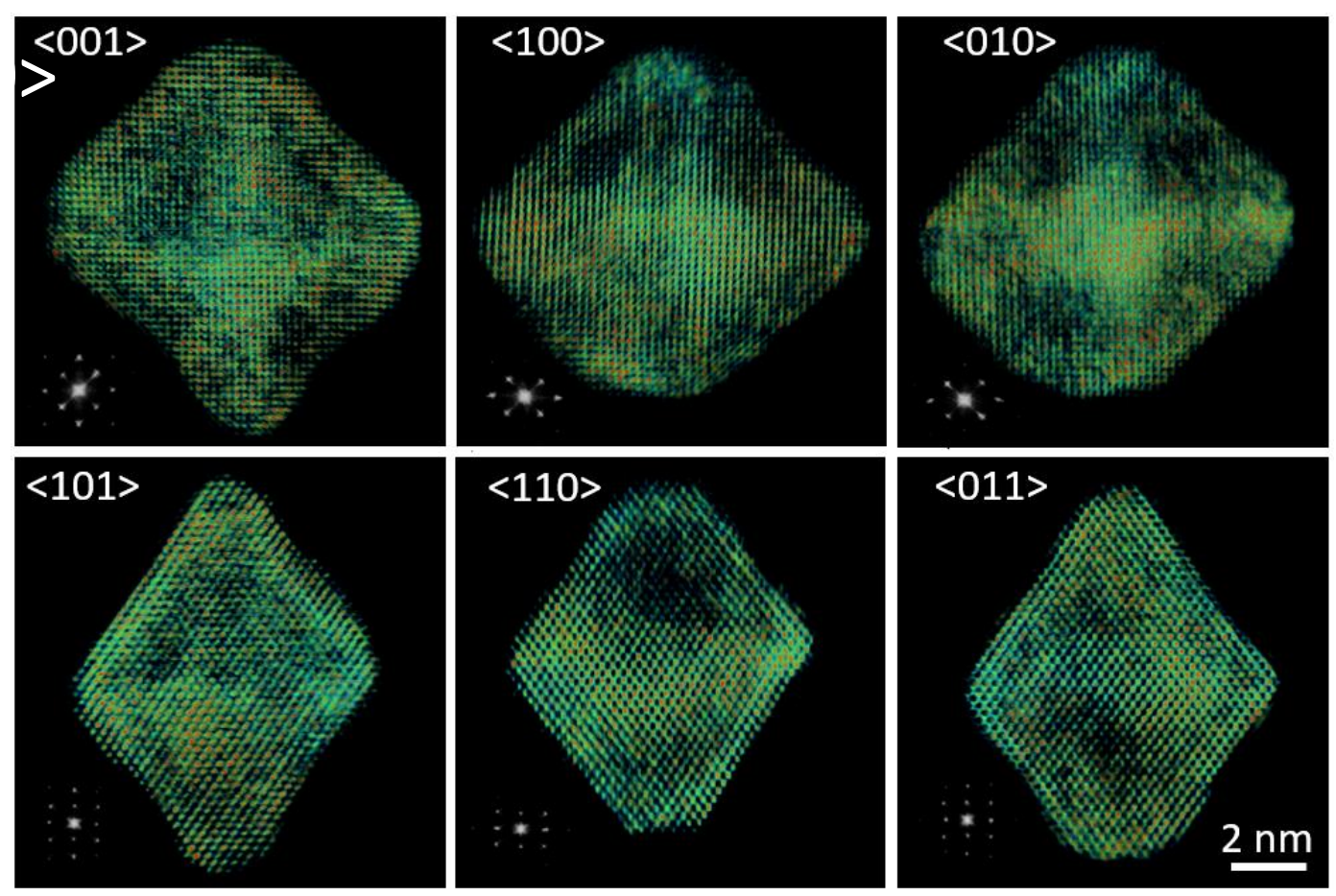

Fig. 1. Volume-renderings of a 3D reconstruction at different orientations. The low left insets show the projected 3D FFT of the reconstruction.
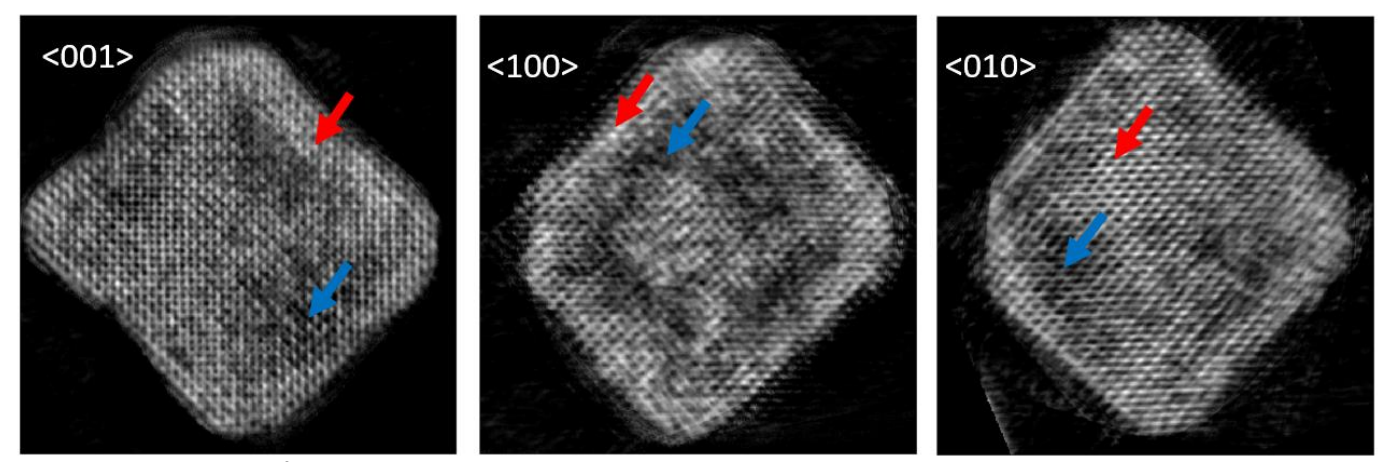

Fig. 2. Three central $1.7 \AA$ thick slices of the $3 \mathrm{D}$ reconstruction along the $\langle 001\rangle,\langle 100\rangle$ and $\langle 010\rangle$ direction, showing clear atom structures. Pt, Ni and Mo can be distinguished by the intensity contrast, and segmentation of $\mathrm{Ni}$ atoms (blue arrows) and $\mathrm{Pt}$ atoms (red arrows) is observed. 